\title{
Laser Embossing of Self-Organized Nanostructures into Metal Surfaces by KrF Laser Irradiation
}

\author{
Martin EHRHARDT, Pierre LORENZ, Marc TEICHMANN, Jan LORBEER, Frank FROST, Klaus ZIMMER \\ Leibniz-Institut für Oberflächenmodifizierung e. V., Permoserstr. 15, 04318 Leipzig, Germany \\ E-mail: klaus.zimmer@iom-leipzig.de
}

\begin{abstract}
In the recent decades the importance of nanostructured surfaces has rapidly grown due to the large range of applications of these improved surfaces in the fields of, e.g. optics, biology, and microelectromechanical systems. However, a flexible and low-cost manufacturing process which is suitable for large-scale solid metal surfaces is not introduced yet. Laser microembossing is a nonthermal manufacturing technology which enables the direct fabrication of three-dimensional micro- and nanostructures. In the present study nanostructures with periods down to $82 \mathrm{~nm}$ were successfully replicated from a fused silica master mould into a copper foil surface by laser embossing. The replicated structures were examined by atomic force microscopy. The results show that the replicated structures have a high resolution and the shape of the replicated structures is similar to the master mould.
\end{abstract}

DOI:10.2961/jlmn.2013.01.0017

Keywords: laser microembossing, nanostructure, mould-based nanoforming, metal sheet forming, laser shock wave

\section{Introduction}

In recent decades, the patterning of surfaces with nanostructures is of rapid growing interest. Beside basic research significant efforts have been done to develop lowcost and flexible surface structuring techniques at the nanometer scale. Typical techniques for nanopatterning of surfaces are electron beam lithography and EUV lithography. However, these techniques require complex equipment and vacuum conditions. More suitable for low-cost nanopatterning of large areas are nanoimprint techniques. With these techniques large-area nanopatterning with structures less than $10 \mathrm{~nm}$ has been demonstrated [1]. However, for patterning of materials like silicon and metals nanoimprint techniques are not appropriated and pattern transfer of resist masks by etching processes such as reactive ion beam etching is necessary. Using laser pulses for melting the target material, the replication of such nanostructures by the so-called laser-assisted direct imprinting (LADI) was demonstrated [2]. With this technique the surface of the work piece is irradiated and melted by a laser pulse and subsequently imprinted using a transparent mould. A resolution of $10 \mathrm{~nm}$ could be demonstrated by patterning nanostructures onto a silicon surface.

Another replication method for metals is laser embossing [3-5]. Laser embossing is a process which is commonly used for microforming of thin metal foils. The process bases on laser pulses which generate a high pressure, e.g. by ablation of an auxiliary material which is in a close contact to the metal foils. The laser-generated high pressure inducing shock waves within the metal foils are causing plastic deformations. Using a structured mould form the metal foils are deformed into the mould and take the shape of the mould. Because the laser pulses are absorbed in the auxiliary material the metal foils are protected from thermal effects; so laser embossing is an almost non-thermal process. Laser embossing on micrometer scale was extensively in- vestigated in the papers of, e.g., Cheng G. J. et al. [6-11], Zhou et al. [12] and Liu et al. [4,13]. Usually single-pulse irradiation with extreme powerful lasers is applied for laser embossing. Hence, the embossed area is limited. To overcome these limitations a multi-pulse scanning approach was introduced by Li J., Cheng G. J. [7] and Ehrhardt M. et al. [14] that enables to replicate large areas. Further, the replication into thick work pieces has been demonstrated by a so-called inverse laser embossing process [15].

However, up to now the laser embossing was performed down to the (sub-) micrometer scale [12].

The aim of this paper is to show that the generation of structures in the nanometer scale is possible with laser embossing. The experiments were performed with commercially rolled thin copper foils. The dimensions and the surface topography of the embossed structures in this copper foils were characterized by atomic force microscopy (AFM). The structures of the mould used for the experiments were compared with the embossed structures in the copper foils to investigate the differences between the structures of the mould and the embossed structures in the copper foils.

\section{Experimental set-up}

In this study laser embossing was performed into thin copper foils. The utilized copper foils with a thickness of $6 \mu \mathrm{m}$ were made by rolling and subsequent soft annealing. The surface roughness of the copper foils measured by AFM was about $300 \mathrm{~nm} \mathrm{rms}\left(50 \times 50 \mu \mathrm{m}^{2}\right)$. The atomic force microscopy (Dimension FastScan AFM (Bruker), FastScan A cantilever, nominal tip radius $<5 \mathrm{~nm}$ ) used in the present study was operated in the tapping mode. A sketch of the experimental set-up used in the present study is shown in Figure 1 (top). First, the master mould and thereupon the copper foil were positioned at a vacuum chuck. Both were covered with a $25 \mu \mathrm{m}$ thick polyimide 
foil that sealed the vacuum chuck. After applying vacuum to the chuck the air pressure pushed the polyimide foil, the metal foil, and the master mould tightly together. Now the polyimide foil was laser-irradiated which resulted in laser ablation and high-density plasma formation. The expanding laser-induced plasma caused a high-amplitude pressure pulse that subsequently propagated as shock wave into the copper foil. A KrF excimer laser with a laser wavelength of $\lambda=248 \mathrm{~nm}$ and a pulse duration of $25 \mathrm{~ns}$ was used for the shock wave generation due to laser ablation of the polyimide foil. The laser workstation used for laser embossing furthermore consists of beam shaping and homogenizing optics, an $\mathrm{x}-\mathrm{y}-\mathrm{z}$ positioning stage, and a dielectric attenuator. The applied square laser spot had an almost homogeneous energy distribution over its size of $100 \times 100 \mu \mathrm{m}^{2}$. The laser fluence and the repetition rate were fixed at values of $3.5 \mathrm{~J} / \mathrm{cm}^{2}$ and $100 \mathrm{~Hz}$, respectively.

The pressure which is induced by ablation of the polyimide foil with a laser of a fluence of $3.5 \mathrm{~J} / \mathrm{cm}^{2}$ is approximately $\sim 1$ kbar [16]. Laser scanning was performed to extend the processing area beyond the laser spot size and this enables a large-area laser embossing. A sketch of the beam path above the sample is shown in Figure 1 (bottom). The velocity of the laser beam was fixed at a value of $1.3 \mathrm{~mm} / \mathrm{s}$ and an overlap of $75 \%$ of parallel laser scanning lines was specified. In consequence, 30 laser pulses were applied in average within the laser-scanned area. The laser irradiation conditions were chosen to avoid perforation of the polyimide foil; the remaining polyimide foil had a thickness of a few micrometers. Because of the short pulse duration and the low heat conductivity of the polyimide foil the remaining polyimide foil prevents excessive heat diffusion to the metal foil. Therefore, the laser embossing process is a non-thermal embossing technique. After the embossing process was performed the processed samples were examined by atomic force microscopy.

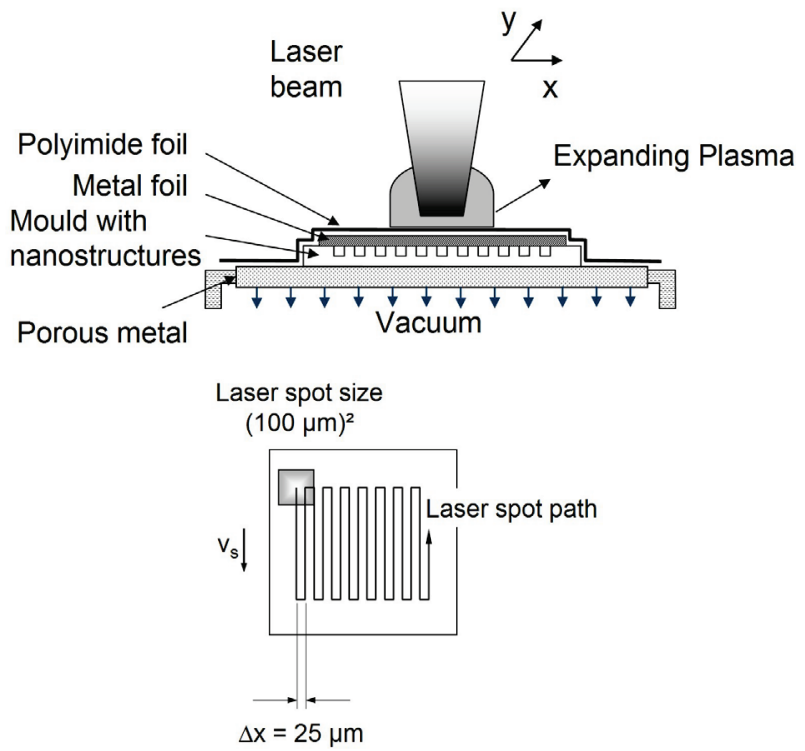

Fig. 1 (top) Sketch of the experimental set-up used for laser embossing of nanostructures. (bottom) Laser spot path applied for large-area replication.

\section{Master mould}

As master moulds self-organized nanopatterned fused silica and silicium samples were applied. Two master moulds featuring substantial different nanopatterns were used in the experiments. The nanostructured surface patterns of the master mould were made by bombardment of the mould surface with low-energy ions. This alternative route for the fabrication of nanostructured surfaces by selforganisation enables the production of very different kinds of surface morphologies. An overview of nanopattern formation by low-energy ion beam erosion is given in $[17,18]$.

To show the capability of transferring very different types of patterns by laser embossing two samples with very different nanostructures were selected. The surface topography of the master moulds was characterized by AFM and is depicted in Figure 2. The master mould (A) has a cauliflower-like appearance whereas the master mould (B) features a grating with a period of approximately $82 \mathrm{~nm}$ on top of a hillocky surface. The features of the patterns are characterized by some statistic broadening as it is characteristic for self-organized surface patterns. In addition, a flat Si wafer with a typical surface roughness of $\sim 1 \mathrm{~nm}$ was used as master mould for laser embossing.

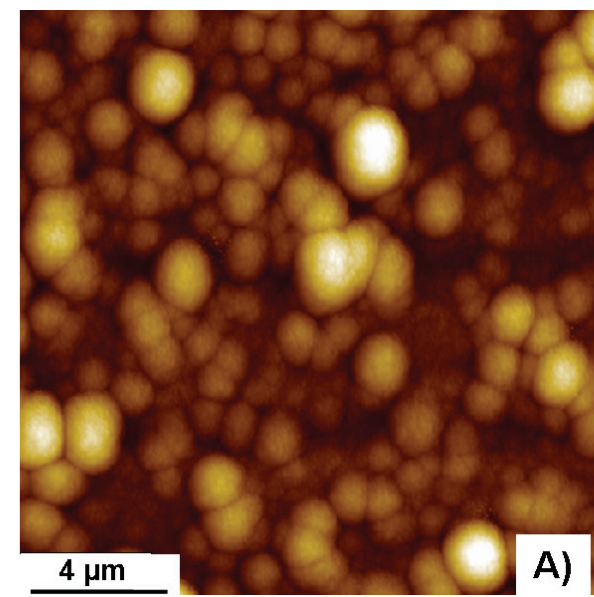

$90 \mathrm{~nm}$
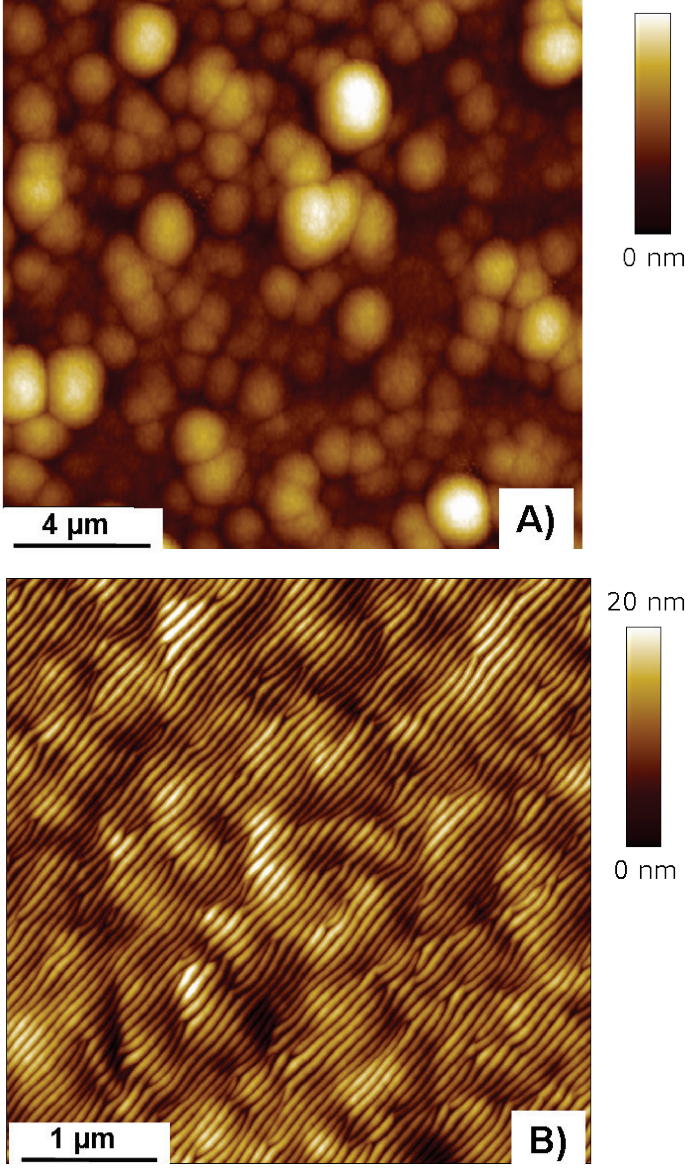

Fig. 2 AFM images of the master moulds used for the embossing process - (top) master mould (A), (bottom) master mould (B).

\section{Results and discussion}

After embossing the copper foil onto the flat silicon wafer the surface of the copper foil was examined with the AFM. From the increased brightness of the foil within the 
laser-embossed areas it was concluded that the original surface roughness of the copper foil had been significantly decreased.
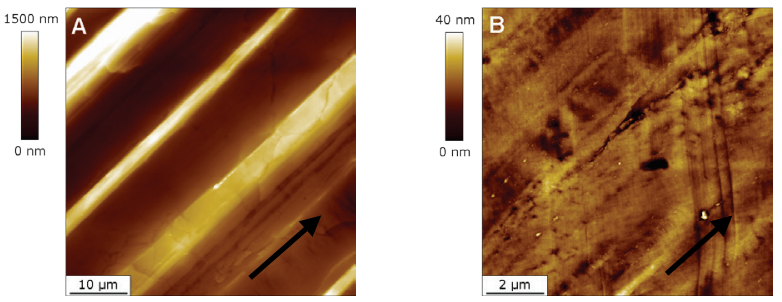

Fig. 3 (A) AFM image of a copper foil before and (B) after embossing with a flat silicon wafer. The arrows show the rolling direction of the foil.

In Figure 3 AFM images of a copper foil surface are shown which is untreated (Figure 3 (A)) and after embossing with a flat silicon wafer (Figure $3(\mathrm{~B})$ )

From the AFM measurement shown in Figure 3 the resulting surface roughness of the copper foil can be calculated. For the untreated copper foil surface roughness values of about $300 \mathrm{~nm} \mathrm{rms}\left(50 \times 50 \mu \mathrm{m}^{2}\right)$ were calculated. After the laser embossing process surface roughness values in the range of $\sim 4 \mathrm{~nm} \mathrm{rms}\left(10 \times 10 \mu \mathrm{m}^{2}\right)$ were obtained. The mainly remaining pattern in the copper foil surface after embossing at the flat master surface is related to the structures from the rolling of the copper foil. The reduction of the surface roughness from $300 \mathrm{~nm}$ rms of an untreated copper surface to $\sim 4 \mathrm{~nm} \mathrm{rms}$ of a laser-formed surface applying a flat silicon master has been observed previously [16]. This significant decrease of the surface roughness should enable the replication of nanostructures by laser embossing into copper foils without its previous smoothing to reduce the surface roughness.

In Figure 4 an AFM image of the inverted surface of a copper foil which was face to face with the master mould (A) during the replication is shown. Basically the morphologies of the master (A) (shown in Figure 2) and the replica (shown in Figure 4) are very similar especially when considering the statistical distribution of the pattern characteristics. However, the replicated features lock slightly blurred and seem to have a minor P-V value compared to the master. The histograms of the heights of the master mould and the replicated sample shown in Figure 4 (bottom) allow the comparison of their height distribution. The height distribution of the master mould and the inverse structures of the laser-embossed replica fit well except the reduced height of deeper structures. In general, a statistic reduction of the height distribution can be supposed. Hence a very proportional replication cannot be simply expected.
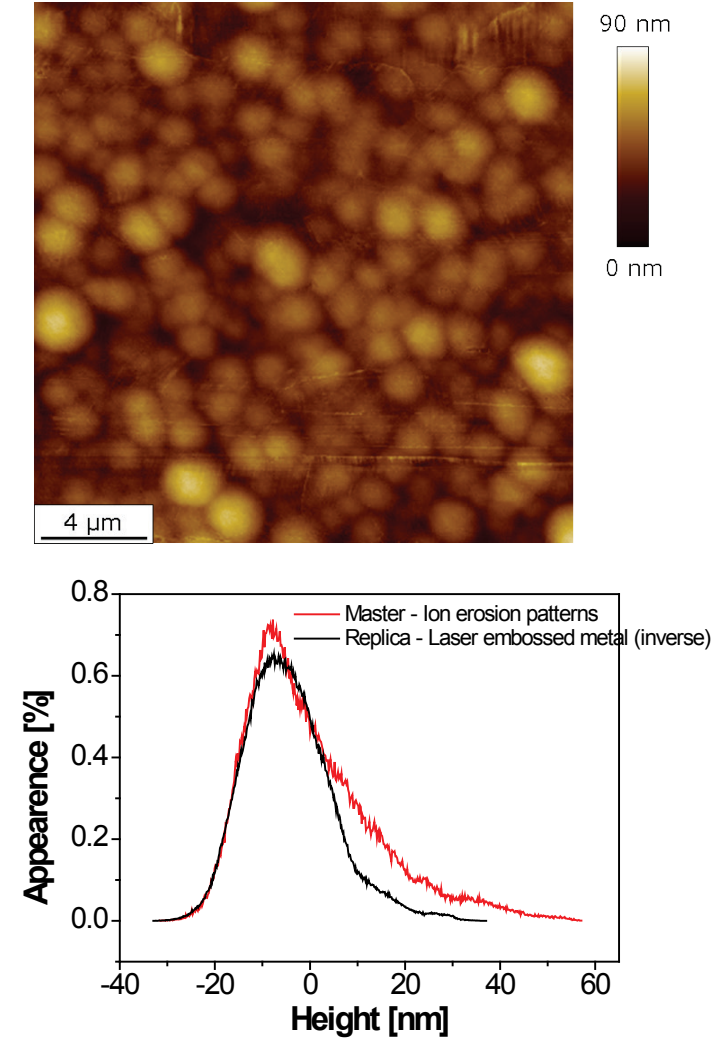

Fig. 4 (top) Inverse AFM image of structures which were replicated from master mould (A) into a copper foil. (bottom) The height distributions of the master and the replica are of the same sample but not of the same area.

In Figure 5 the AFM image shows the replicated structure from the master mould (B), the nanometer-sized grating patterns and the hillock structures, and, in addition, some irregular pattern. The origin of these irregular structures due to the embossing process is not fully understood. One reason might be remaining scratches of the foil surface. Further possible reasons are particles whose shapes are embossed as well. However, comparing the long scratches shown in the AFM image of Figure 3 (A) with the remaining pattern at Figure 3 (B) the similarities support the suggestion that these are related to the rolling patterns. Hence, the surface topography can be assumed to be a superposition of patterns from embossing a flat surface with the replicated nanostructures of the master.

To analyze whether the main structure of the replica correlates with the structures in the master mould and to quantify the quality of the embossing process frequency spectra were calculated from the AFM image of the master and the replicated structures. The frequency spectra of the master mould (B) and of the replication from master mould (B) are shown in Figure 5 (bottom). There it can be seen that the main peak of the master mould corresponds to a structure period of $\lambda=82 \mathrm{~nm}$ and the FWHM (Full Width at Half Maximum) is $\sim 50 \mathrm{~nm}$. The replicated structures have an average period of $\lambda=87 \mathrm{~nm}$ and a FWHM of $\sim 41 \mathrm{~nm}$. Hence, the structure size/period is only slightly changed by the replication of the master mould patterns into the copper foil surface.

However, the amplitude of the patterns changes due to the laser embossing. The replica features a much smaller 
amplitude compared to the master patterns. Although this might be the result of the incomplete embossing over the size of the AFM image also the local P-V values of the grating show that the height of the master pattern (10 to $13 \mathrm{~nm}$ ) is greater that that of the replica (7 to $10 \mathrm{~nm}$ ).

The reduction of the heights between the master structures and the replicas seems to be a general effect because it appears by the replication of the master moulds (A) and (B).
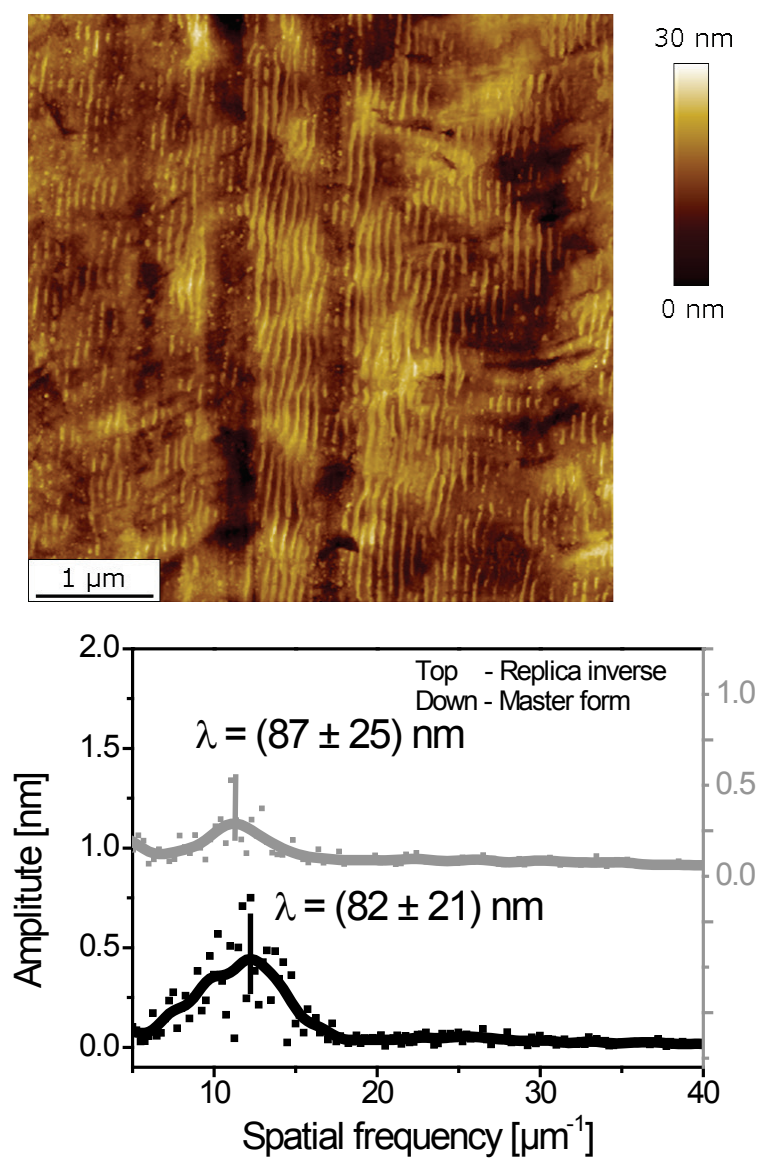

Fig. 5 (top) AFM image of the replicated structures in the copper foil. The used master mould was master mould (B). (bottom) Frequency spectra of the master mould (B) and the replicated structures in the copper foil (top).

The reasons for the lower height of the replicated structures than of the master structures are not clear yet. One reason could be that the replication process comprises both elastic and plastic deformations. The elastic part of the deformation due to the replication process relaxes after the pressure load is removed. The plastic part of the deformation is retained after laser embossing and accounts for the depth of the embossed pattern. Hence, to achieve a proportional pattern transfer by laser embossing with a desired height of the replica patterns, e.g. for some kind of application, the master structures height might be slightly higher.

From this result it can be assumed that much smaller structures can be replicated from a master structure into a work piece by laser embossing. The comparison of the found result of the laser embossing process with results of "classical" mechanical embossing in the sub-micrometer range is difficult because of the different time scales in which the two processes occur. Additionally, only a few papers were reported which investigated embossing on the nanometer scale, e.g. [19-21]. In the work of Ke Chen et al. [20] mechanical micro- to nanoscale moulding of aluminium was investigated. Although this study is not based on copper it gives an indication of the required pressure for the embossing process. From this report it can be concluded that the required characteristic moulding pressure increases with a decreasing embossed pattern height. For the investigated aluminium in the study of $\mathrm{Ke}$ Chen et al. characteristic moulding pressures of about $5.6 \mathrm{kbar}$ for structure widths of $550 \mathrm{~nm}$ have been measured. Taking into account that the yield strength and the Young's modulus of copper are even higher than that of aluminum it can be assumed that the characteristic moulding pressure is higher for "classical" mechanical embossing of copper. As mentioned in the experimental section for the laser embossing process used peak pressures as high as $1 \mathrm{kbar}$ can be expected, which are lower than the characteristic moulding pressures which are applied for "classical" mechanical embossing.

\section{Conclusion}

In conclusion, nanostructures can be transferred from a master mould into a copper foil by laser embossing using a laser scanning approach. The replicated structures have a lateral resolution well within the nanometer range.

Patterns with a height of some nanometers can be replicated. The reduction of the pattern height at laser embossing is probably due to the elastic deformation during embossing. In consequence, for achieving a specific embossing depth at given laser embossing parameters the pattern height of the master should be slightly increased.

The results show that with laser embossing low-cost and large-scale patterning of thin metal foils can be achieved. With further improvements of the laser embossing process this technique has the potential to become an important fabrication method in the field of surface nanoengineering.

\section{Acknowledgments}

The authors are deeply indebted to E. Salamatin for the careful reading of the manuscript.

\section{References}

[1] S. Y. Chou, P. R. Krauss, W. Zhang, L. J. Guo, L. Zhuang, J. Vac. Sci. Technol., B 15 (1997) 2897.

[2] B. Cui, C. Keimel, S. Y. Chou, Nanotechnology 21 (2010).

[3] M. Zhou, Y. K. Zhang, L. Cai, J. Appl. Phys. 91 (2002) 5501.

[4] H. X. Liu, Z. B. Shen, X. Wang, H. J. Wang, M. K. Tao, Applied Surface Science 256 (2010) 4687.

[5] H. Wielage, F. Vollertsen, Journal of Materials Processing Technology 211 (2011) 953.

[6] J. Li, H. A. Gao, G. J. Cheng, Journal of Manufacturing Science and Engineering-Transactions of the Asme 132 (2010) 10.

[7] J. Li, G. J. Cheng, J. Appl. Phys. 108 (2010). 
[8] H. Gao, C. Ye, G. J. Cheng, Journal of Manufacturing Science and Engineering-Transactions of the Asme 131 (2009) 11.

[9] H. Gao, G. J. Cheng, J. Appl. Phys. 109 (2011) 11.

[10] H. Gao, G. J. Cheng, Journal of Microelectromechanical Systems 19 (2010) 273.

[11] G. J. Cheng, D. Pirzada, Z. Ming, J. Appl. Phys. 101 (2007) 7.

[12] M. Zhou, T. Huang, L. Cai, Appl. Phys. A-Mater. Sci. Process. 90 (2008) 293.

[13] H. X. Liu, Z. B. Shen, X. Wang, H. J. Wang, J. Appl. Phys. 106 (2009) 4.

[14] M. Ehrhardt, P. Lorenz, K. Zimmer, Lasers in Engineering in press (2012).

[15] M. Ehrhardt, P. Lorenz, F. Frost, K. Zimme, Physics Procedia (submitted).

[16] A. D. Zweig, V. Venugopalan, T. F. Deutsch, J. Appl. Phys. 74 (1993) 4181.

[17] F. Frost, B. Ziberi, A. Schindler, B. Rauschenbach, Appl. Phys. A-Mater. Sci. Process. 91 (2008) 551.

[18] F. Frost, A. Schindler, F. Bigl, Phys. Rev. Lett. 85 (2000) 4116.

[19] A. I. M. Greer, K. Seunarine, A. Z. Khokhar, X. Li, D. A. J. Moran, N. Gadegaard, Phys. Status Solidi AAppl. Mat. 2091721.

[20] K. Chen, W. J. Meng, F. H. Mei, J. Hiller, D. J. Miller, Acta Mater. 591112.

[21] C. W. Hsieh, H. Y. Hsiung, Y. T. Lu, C. K. Sung, W. H. Wang, J. Phys. D-Appl. Phys. 40 (2007) 3440.

(Received: September 26, 2012, Accepted: January 21, 2013) 